\title{
Über das Vorhandensein des Treponema pallidum im Gehirn der progressiven Paralyse.
}

\author{
Von \\ J. W. Moore, M. D. \\ (Aus dem Central Islip State Hospital, Central Islip, New York.) \\ Mit 1 Tafel. \\ (Eingegangen am 1. April 1913.)
}

In einer Anzahl von Paralytikergehirnen, von welchen der Verfasser Schnitte anfertigte und färbte, um die Frage nach der Anwesenheit oder dem Fehlen der Spirochaeta pallida im Gehirn der Paralyse zu entscheiden, ist es gelungen, diese Organismen tatsächlich nachzuweisen. Dieselben wurden zuerst von Nog u chi im Rockefeller Institute gesehen. Letzterer hatte den Verfasser gebeten, ihm von seinen Präparaten zu schicken, weil er sich überzeugen wollte, ob etwa die Spirochaeta in körniger Form im Paralytikergehirn zu finden sei. Ein kurzer Bericht über diese Befunde wurde im Februar dieses Jahres veröffentlicht' ${ }^{1}$ ).

Der Zweek der vorliegenden Mitteilung ist es, den Befund des Vorhandenseins des Treponema pallidum (Spirochaeta pallida) im Gehirne von Paralytikern zur Kenntnis der Psychiater und Neurologen zu bringen. Einige Bemerkungen über die Gründe, welche zu dieser Arbeit Veranlassung gaben, sowie über die Art und Weise der Ausführung dürften hier am Platze sein.

Im Jahre 1910, in der Review of Neurology and Psychiatry (Edinburgh), veröffentlichte der Verfasser einèn Aufsatz, der einen Überblick äber die Literatur gab und einige persönliche Erfahrungen betreffs der Syphilis-Paralysefrage anfügte. Eine der Schlußfolgerungen ist: ,General paralysis must still be regarded as a metasyphilitic disease rather than true syphilis", und ich denke, das war wohl damals und ist noch jetzt die Ansicht der meisten Psychiater. Die Hauptstütze dieser Annahme lag darin, daß die syphilitischen Organismen bei der Paralyse nie gefunden worden waren, weder im Géhirne, noch in den übrigen Organen, noch in der Aorta. Nichtsdestoweniger muß zugegeben werden, daß eine gewisse Anzahl von Forschern nicht überzeugt waren, daß die Paralyse doch nicht eine Art der wahren Syphilis sei. Unter den Klini-

1) Noguchi and Moore, Journ. of Experim. Med. 17, 2. 1913. 
kern möchte ich in diesem Zusammenhange Da na erwähnen; unter den Histologen Dunlap, dessen Ansicht sich besonders auf seine Studien an Utbergangsfällen von Spätsyphilis des Nervensystems und progressiver Paralyse stützt; und schließlich unter den Serologen muß Pla u t erwähnt werden, welch letzterer wenigstens auf Grund seiner Untersuchungen sich überzeugte, daß die Spirochaeta irgendwo in Organismen der Paralytiker vorhanden sein müsse, da auf eine andere Weise das Komplementablenkungsphänomen nicht erklärt werden könnte.

Obgleich noch ein Anhänger der metasyphilitischen Lehre, die jedoch keinen befriedigenden Abschluß bedeutete, sondern bloß als eine vorläufige Formulierung konnte angesehen werden, war der Verfasser doch der Ansicht, daß zur weiteren Klärung der Frage vor allem zwei Dinge nötig seien, nämlich eine sorgfältige Durchsuchung von Paralytikergehirnen nach dem Treponema pallidum, und zweitens eine experimentelle Einimpfung von Material paralytischer Gehirne in die Hoden von Kaninchen und den Subduralraum von Affen, um zu sehen, ob dadurch die histologischen Veränderungen von Syphilis resp. Paralyse hervorgebracht werden können. Soviel mir bekannt, sind solche Experimente noch nicht unternommen worden. Ein Umstand, der mehr als irgendein anderer die Durchforschung nach der Spirochaeta im Gehirne zu rechtfertigen schien, waren die Verhältnisse bei der Trypanosomiasis (Gambiense), einer Krankheit, die bekanntlich große Analogie zu Syphilis aufweist. Sie ist verursacht durch ein Protozoon, nahe verwandt mit der Treponemagruppe, sie weist frühe exanthematöse und febrile Krankheitserscheinungen mit späten, tötlichen Nervenstörungen auf (Schlafkrankheit). Das histologische Bild im Gehirne ist dem der Paralyse sehr ähnlich; jedoch bei Schlafkrankheit kann das Trypanosoma leicht im Gehirne und Liquor gefunden werden. Sollte es deshalb nicht auch möglich sein, das Treponema pallidum im paralytischen Gehirne zu finden?

Infolge seiner Tätigkeit als Pathologe im Central Islip State Hospital hatte der Verfasser eine ziemlich große Anzahl paralytischer Gehirne zur Verfügung, und von diesen bildeten 70 Gehirne von klinisch und anatomisch sichergestellten Fällen von Paralyse den Gegenstand der Untersuchung. Die Einzelheiten der Technik der Färbung will ich übergehen. Es genüge, zu sagen, daß die Levaditi - Silber - Methode angewandt wurde, mit einigen Modifikationen, die möglicherweise von Wichtigkeit waren, möglicherweise auch nicht. Die Mehrzahl der Stückchen wurde dem frontalen Teile der Gehirne entnommen, mit der Absicht, solche Teile zu untersuchen, wo der Krankheitsvorgang am intensivsten war, soweit dies durch die Verdickung der Pia und die Atrophie der Gehirnsubstanz ersichtlich war. Gegenwärtig ist es die Uberzeugung des Verfassers, daß die gewöhnliche Levaditi-Technik genügt für den Zweck, 
doch ist diese Färbung kapriziös und manche Schnitte sind untauglich für die Untersuchung auf Treponemata, entweder wegen zu großer Affinität der Neurogliafasern für das Silber oder wegen körnigen Niederschlages des Metalles durch das ganze Gewebe. Es war deshalb nur durch die Untersuchung einer ganz beträchtlichen Anzahl von Exemplaren möglich, Präparate zu erhalten, in denen Nerven- und Neurogliafasern keinen oder nur geringen Niederschlag aufwiesen. Ein Versuch, eine Methode ausfindig zu machen, die immer gleichgute Resultate ergab, ist bisher noch erfolglos geblieben. Das Material des Verfassers gab ungewöhnlich günstige Erfolge der Färbung in vielen Exemplaren. Im ganzen konnte die Anwesenheit der Spirochaeten in 12 Fällen gezeigt werden; in ungefähr der Hälfte von diesen waren sie ziemlich zahlreich und nicht schwierig $\mathrm{zu}$ finden, in anderen wiederum waren sie außerordentlich selten. Es ist interessant und bedeutungsvoll, daß sie, trotz sorgfältiger Nachforschung, weder in cer Pia noch in den Wänden der großen Gefäße gefunden wurden, wo man doch erwarten sollte, sie anzutreffen, sondern tief in dem Nervergewebe. Ihre Bewegungsfähigkeit und Durchdringungskraft sind bekannt durch Dunkelfeldstudien und durch Nog u chis Kulturexperimente; und es war offenbar infolge dieser Fähigkeiten, daß sie den Weg fanden, zu den Regionen, in denen sie angetroffen wurden. Wo sie vorkommen, trifft man sie fast immer in den Nervzellenschichten der Gehirnrinde an, selten im Marke und nie in der äußeren Neurogliaschicht, soweit die Beobachtung an den obigen Fällen in Betracht kommt.

Die zwei hauptsächlichsten Fragen, die aufgeworfen werden könnten in bezug auf den Nachweis von Spirochäten im Paralytikergehirn betreffen die Sicherheit der Diagnose der Paralyse und die Identität des Organismus mit dem Treponema pallidum. Es ist wohl kaum nötig, photographischen Beweis für das erstere zu erbringen. Das histologische Bild der Paralyse war in allen Fällen vollständig sicher und das einzige mögliche Anzeichen von komplizierender Gehirnsyphilis, im engeren Sinne des Wortes, war ein leichter Grad von augenscheinlich chronischer Endarteritis in einigen Fällen. Betreffs des Organismus können wir bestimmt behaupten, daß es eine Spirochaeta ist und daß sie die Morphologie der Pallida aufweist. Es ist möglich, ja wahrscheinlich, daß durch Einimpfungen und Kulturen der Beweis erbracht wird, daß es sich hier in der Tat um den wahren syphilitischen Organismus handelt. Die einzige andere Möglichkeit ist, daß es sich um eine der Mundspirochäten handelt. Die Spirochaeta dentium ist ein zarter, schlanker Organismus, ähnlich der Pallida, und zeigt die gleiche Anzahl von Wellungen. Diese Spirochaeta jedoch kommt fast ausschließlich nur um die Zähne herum vor, und diejenige, die sich im Schlund befindet und von welcher Gerber bewiesen hat, daß sie für die meisten Fälle von Vincents Angina und 
sogar Skorbut verantwortlich ist, ist die Spirochaeta bucealis. Im Aussehen ist sie jedoch völlig verschieden, sie ist dick und hat nur 3-5 flache Wellungen. In Anbetracht ihres Aufenthaltsortes konnte diese letztere am leichtesten das Gehirn erreichen, obwohl dies äußerst unwahrscheinlich ist, und es ist kaum denkbar, daß sie, selbst durch den Vorgang der Erhärtung und Färbung, ihre Form zu derjenigen, die wir in diesen Exemplaren sahen, ändern könnte.

Einige Bemerkungen betreffs der Bedeutung der Entdeckung des syphilitischen Organismus im Gehirne bei Paralyse sind hier gewiß am Platze. Die Lokalisierung und Verbreitung des Organismus, soweit dies aus den 12 Fällen ersichtlich ist, sind genügend übereinstimmend mit der Verteilung des paralytischen Prozesses und gewähren deshalb eine zufriedenstellende Erklärung für die letztere. Der Unterschied zwischen dem Krankheitsvorgange bei Gehirnsyphilis und demjenigen bei Paralyse ist leichter verständlich gemacht. Es ist nicht schwierig, im ersteren Falle sich eine lokalisierte syphilitische Erkrankung in der Hirnhaut vorzustellen, mit oder ohne Endarteritis, jedoch mit Erhaltung einer schützenden Barriere gegen das Eindringen in die Gehirnsubstanz, während im letzteren Falle eine allgemeine Verbreitung des Organismus anzunehmen ist infolge verschiedener Umstände, sei es durch Alkohol, Erblichkeit oder durch die Prädilektion einer gewissen Art der Organismen für das Nervengewebe usw. Die Gegenwart des Treponema, welche in wenigstens einem Falle in tätigem Zustande zu sein schien, gibt uns den Grund für die serologischen Befunde bei der Paralyse, und durch ihren tiefen Sitz und weite Verbreitung ist die Unzulänglichkeit der antisyphilitischen Behandlung erklärlich, wenn man den Umstand im Lichte der Experimente mit vitaler Färbung und die Tatsache, daß der Farbstoff das Nervengewebe nicht erreicht, betrachtet. Paralytische Anfälle mögen nun als Folgen von weiterem Eindringen des Organismus angesehen werden, und dies ist wohl wahrscheinlicher, als sie auf unbestimmte Stoffwechselstörungen oder Kongestionen im Gehirne zurückzuführen. Die Leukocytose, die diese Erscheinungen begleitet, ist vollkommen in Einklang mit dieser Erklärung. In unserer Auffassung cer Ätiologie der Paralyse sind nun Theorien über Stoffwechselstörungen, Überproduktion der syphilitischen Antikörper, Mangel an Lecithin usw. unnötig geworden.

Jedoch bleibt unsere Stellung zu der Sypbilis-Paralysefrage in gewissen Beziehungen unverändert. Unsere Annahme ,ohne Syphilis keine Paralyse" erhält keine weitere Stütze, bis es möglich ist, den Organismus in allen Fällen von Paralyse zu demonstrieren. Wir haben immer noch keine Erklärung für das lange Intervall, cas sich zwischen syphilitische Infektion und Paralyse einschiebt. Daß die Organismen in dieser Zeit im Ruhezustand liegen können, scheint durch einen Fall, den Eichelberg 
und Pförtner erwähnen, bewiesen. Es handelt sich hier um einen Mann, der 10 Jahre vor der Untersuchung Syphilis hatte, keine Komplementablenkung im Blutserum aufweist, dagegen $1 \frac{1}{2}$ Jahre nach der Untersuchung eine Paralyse entwickelte und zugleich eine positive Wassermann-Reaktion im Blutserum zeigte. Für die weit verbreiteten Degenerationserscheinungen des Nervensystems, die keine Beziehungen zu dem chronischen Entzündungsvorgang haben, müssen wir wohl einen anderen Grund als die Gegenwart des Treponema suchen, es sei denn, daß wir annehmen, letzteres habe einen chemischen Einfluß oder bringe chemische Veränderungen durch seine Desintegrierung hervor. Die Frage, ob Paralyse wahre Syphilis sei oder nicht, bleibt daher offen; jedoch der wichtigste Punkt, der im Widerspruch hierzu stand, ist nun entfernt. 